Copyright (C) 2013 IEEE. Personal use of this material is permitted. Permission from IEEE must be obtained for all other uses, in any current or future media, including reprinting/republishing this material for advertising or promotional purposes, creating new collective works, for resale or redistribution to servers or lists, or reuse of any copyrighted component of this work in other works. 


\title{
Arbitrary pole placement by state feedback with minimum gain
}

\author{
Robert Schmid, Lorenzo Ntogramatzidis, Thang Nguyen and Amit Pandey
}

\begin{abstract}
We consider the classic problem of pole placement by state feedback. We offer an eigenstructure assignment algorithm to obtain a novel parametric form for the pole-placing gain matrix that can deliver any set of desired closed-loop eigenvalues, with any desired multiplicities. This parametric formula is then exploited to introduce an unconstrained nonlinear optimisation algorithm to obtain a gain matrix that delivers the desired pole placement with minimum gain.
\end{abstract}

\section{INTRODUCTION}

We consider the classic problem of repeated pole placement for linear time-invariant (LTI) systems in state space form

$$
\dot{x}(t)=A x(t)+B u(t)
$$

where, for all $t \in \mathbb{R}, x(t) \in \mathbb{R}^{n}$ is the state and $u(t) \in \mathbb{R}^{m}$ is the control input, and $A$ and $B$ are appropriate dimensional constant matrices. We also assume that $B$ has full column-rank, and that the pair $(A, B)$ is reachable. We let $\mathscr{L}=\left\{\lambda_{1}, \ldots, \lambda_{v}\right\}$ be a self-conjugate set of $v \leq n$ complex numbers, with associated algebraic multiplicities $\mathscr{M}=\left\{m_{1}, \ldots, m_{v}\right\}$ satisfying $m_{1}+\cdots+m_{v}=n$. The problem of exact pole placement (EPP) by state feedback is to find a real gain matrix $F$ such that the closed-loop matrix $A+B F$ has eigenvalues given by the set $\mathscr{L}$ with multiplicities given by $\mathscr{M}$, i.e., such that $F$ satisfies the equation

$$
(A+B F) X=X \Lambda,
$$

where $\Lambda$ is a $n \times n$ Jordan matrix obtained from the eigenvalues of $\mathscr{L}$, including multiplicities, and $X$ is a matrix of closed-loop eigenvectors of unit length. The matrix $\Lambda$ can be expressed in the Jordan (complex) canonical form

$$
\Lambda=\operatorname{diag}\left\{J\left(\lambda_{1}\right), J\left(\lambda_{2}\right), \ldots, J\left(\lambda_{v}\right)\right\}
$$

where each $J\left(\lambda_{i}\right)$ represents a Jordan matrix for the eigenvalue $\lambda_{i}$ of order $m_{i}$, and may be composed of up to $m$ mini-blocks ${ }^{1}$

$$
J\left(\lambda_{i}\right)=\operatorname{diag}\left\{J_{1}\left(\lambda_{i}\right), J_{2}\left(\lambda_{i}\right), \ldots, J_{g_{i}}\left(\lambda_{i}\right)\right\},
$$

Partially supported by the Australian Research Council under the grant FT120100604.

Robert Schmid is with Department of Electrical and Electronic Engineering, University of Melbourne, Australia. E-mail: rschmideunimelb.edu.au

L. Ntogramatzidis is with the Department of Mathematics and Statistics, Curtin University, Perth, Australia. E-mail: 1.ntogramatzidis@curtin.edu.au

Thang Nguyen is with Department of Engineering, University of Exeter, UK. E-mail: T.Nguyen-Tiendexeter.ac.uk

Amit Pandey is with Department of Mechanical and Aerospace Engineering, University of California, San Diego, USA. E-mail: appandey@ucsd.edu

${ }^{1}$ Each $J\left(\lambda_{i}\right)$ is composed of up to $m$ mini-blocks because, as will be mentioned in the sequel, when the pair $(A, B)$ is reachable, the dimension of $\operatorname{ker}\left[\begin{array}{lll}A-\lambda I & B\end{array}\right]$ is equal to $m$ for any $\lambda \in \mathbb{C}$. where $g_{i} \leq m$ and for each $k \in\left\{1, \ldots, g_{i}\right\}$, we denote by $p_{i, k}$ the order of the Jordan block $J_{k}\left(\lambda_{i}\right)$. It is well-known that arbitrary multiplicities can be assigned, but the possible orders of the associated Jordan structures are constrained by the system controllability indices (or Kronecker invariants) as established in the celebrated Rosenbrock theorem, [1]. We use $\mathscr{P} \triangleq\left\{p_{i, j} \mid 1 \leq i \leq v, 1 \leq j \leq g_{i}\right\}$ to denote the order of each Jordan mini-block $J_{j}\left(\lambda_{i}\right)$, and we assume without loss of generality that for each $i$, these are in descending order $p_{i, 1} \geq p_{i, 2} \geq \cdots \geq p_{i, g_{i}}$. If $\mathscr{L}, \mathscr{M}$ and $\mathscr{P}$ satisfy the conditions of the Rosenbrock theorem, we say that $\mathscr{L}, \mathscr{M}$ and $\mathscr{P}$ define an admissible Jordan structure.

In order to consider optimal selections for the gain matrix, it is important to have a parametric formula for the set of gain matrices that deliver the desired pole placement, and numerous such parameterisations have appeared in the literature in the past three decades. Kautsky et al. [2] introduced a parametric form involving a $\mathrm{QR}$-factorisation for matrix $B$ and a Sylvester equation for $X$, but required $\Lambda$ in (2) to be a diagonal matrix. In particular this requires the desired multiplicities to satisfy $m_{i} \leq m$ for all $i \in\{1, \ldots, v\}$. This limitation is inherited by the MATLAB ${ }^{\circledR}$ routine place.m that is based on [2]. The pole-placement methods of Byers and Nash [3] and Tits and Yang [4] similarly employed the parametric form of [2] and likewise cannot assign poles with multiplicity greater than the rank of $B$. In the recent paper [5] it is also assumed that the multiplicities of the closed-loop eigenvalues is at most equal to $m$.

Other parameterisations have been presented in the literature that do not impose a constraint on the multiplicity of the eigenvalues to be assigned. Bhattacharyya and de Souza [6] gave a procedure for obtaining the gain matrix by solving a Sylvester equation in terms of an $n \times m$ parameter matrix, provided the closed-loop eigenvalues do not coincide with the open loop ones. Fahmy and O'Reilly in [7] presented a parametric form in terms of the inverses of the matrices $A-\lambda_{i} I_{n}$ (where $I_{n}$ denotes the $n \times n$ identity matrix), which also required the assumption that the closed-loop eigenvalues were all distinct from the open loop ones.

More recently, Chu, [8] revisited the parametric formula of [2] for the case where $\Lambda$ was any admissible Jordan matrix, and obtained a parametrisation for the pole placing matrix $F$ by using the eigenvector matrix $X$ as a parameter. Ait Rami et al. [9] proposed a parametric form in terms of the solution to a Sylvester equation involving the real Jordan form of the desired closed-loop eigenvalues and an arbitrary $n \times n$ matrix parameter. These formulations allow for repeated eigenvalues and does not require the closed-loop poles to be different from the open-loop poles. On the other 
hand, maximum generality in these parametric formulae has been achieved at the expense of efficiency. Where methods [2]-[7] all employed parameter matrices of dimension $m \times n$, the parameter matrices in [8] -[9] have dimension $n \times n$.

The aim of this paper is to offer a parameterisation for the pole-placing feedback that combines the generality of [8]-[9] with the efficiency that comes from an $m \times n$ dimensional parameter matrix. Inspired by the Klein-Moore algorithm [10], we offer a parametric form for the pole-placing gain matrix $F$ to obtain any desired Jordan structure that satisfies the structural constraints imposed by the Rosenbrock theorem in terms of the Kronecker invariants, without any additional requirement on their multiplicity or on non-overlap with the open-loop eigenstructure. The method proposed in this paper obtains the matrix $X$ by building the Jordan chains starting from the selection of eigenvectors from the kernel of the matrix pencils $\left[\begin{array}{lll}A-\lambda_{i} I_{n} & B\end{array}\right]$, and thus avoids the need for matrix inversions, or the solution of matrix equations.

We next consider the minimum gain exact pole placement problem (MGEPP), which involves solving the EPP problem and also obtaining the feedback matrix $F$ that has the smallest gain and best accuracy. Tam and Lam [11] addressed the MGEPP problem by posing an unconstrained nonlinear optimisation problem, in which they sought to minimise the Frobenius norm of the pole-placing matrix $\|F\|_{\text {FRO }}$ by gradient search methods, to yield a locally optimal gain matrix. However their problem formulation was limited to the case where all the desired closed-loop poles were distinct, and did not coincide with any of the open loop poles. Linnemann [12] addressed the MGEPP problem for the specific case of placing multiple deadbeat modes with minimum Frobenius gain.

In this paper we address the MGEPP problem in a manner similar to that of [11], and pose an unconstrained nonlinear optimisation problem, to be solved by gradient search methods, to yield a locally optimal gain matrix. While [11] required the closed loop poles to all be distinct, the use of the very general parametric formula for the gain matrix presented in this paper will enable us to achieve any desired multiplicities, while also minimising the feedback gain used. We then compare the results against the methods of [9] and [12] and show in two examples that we can obtain repeated pole placement with less gain and superior accuracy of the pole placement.

\section{REPEATED POLE PLACEMENT}

We now revisit the Klein-Moore algorithm [10] and adapt it to give a simple parametric formula for a gain matrix $F$ that solves the pole placement problem, in terms of an arbitrary real parameter matrix. We begin with some definitions and notation. For each $i \in\{1, \ldots, v\}$, we define the matrix

$$
S\left(\lambda_{i}\right) \triangleq\left[\begin{array}{ll}
A-\lambda_{i} I_{n} & B
\end{array}\right]
$$

Since each $S\left(\lambda_{i}\right)$ has $n$ rows and $n+m$ columns, and the pair $(A, B)$ is reachable, the dimension of the kernel of $S\left(\lambda_{i}\right)$ is equal to $m$. We denote by $N_{i}$ a basis matrix for the kernel of $S\left(\lambda_{i}\right)$. It follows that, if $\lambda_{i+1}=\bar{\lambda}_{i}$, then $N_{i+1}$ is given by $N_{i+1}=\bar{N}_{i}$. We let

$$
M_{i} \triangleq\left[\begin{array}{ll}
A-\lambda_{i} I_{n} & B
\end{array}\right]^{\dagger},
$$

where ${ }^{\dagger}$ indicates the Moore-Penrose pseudo-inverse.

For any matrix $X$ we use $X(l)$ to denote the $l$-th column of $X$. If $X$ is a vector or matrix with $n+m$ rows, we define the vectors or matrices $\bar{\pi}\{X\}$ and $\underline{\pi}\{X\}$, obtained by taking the first $n$ and last $m$ rows of $X$, respectively.

Given a set of $v$ self-conjugate complex numbers $\mathscr{L}=$ $\left\{\lambda_{1}, \ldots, \lambda_{v}\right\}$ containing exactly $\sigma$ complex conjugate pairs, we say that $\mathscr{L}$ is $\sigma$-conformably ordered if the first $2 \sigma$ values of $\mathscr{L}$ are complex while the remaining are real, and for all odd $k \leq 2 \sigma$ we have $\lambda_{k+1}=\bar{\lambda}_{k}$. For example, the set $\mathscr{L}=\{10 j,-10 j, 2+2 j, 2-2 j, 7\}$ is 2-conformably ordered. Notice that, since $\mathscr{L}$ is symmetric, we have $m_{i}=$ $m_{i+1}$ for odd $i \leq \sigma$.

Let $\mathscr{L}=\left\{\lambda_{1}, \ldots, \lambda_{v}\right\}$ be $\sigma$-conformably ordered. Let $\mathscr{L}$, $\mathscr{M}$ and $\mathscr{P}$ define an admissible Jordan structure. Let $K \triangleq$ $\operatorname{diag}\left\{K_{1}, \ldots, K_{v}\right\}$, where $K_{i}$ is a real matrix of dimension $m \times m_{i}$, for each $i \geq 2 \sigma, K_{i}$ is a real matrix of dimension $m \times m_{i}$, and for all odd $i \leq 2 \sigma$, we have $K_{i}=\bar{K}_{i+1}$. Further, let each $K_{i}$ matrix be partitioned as

$$
K_{i}=\left[\begin{array}{l|l|l|l}
K_{i, 1} & K_{i, 2} & \ldots & K_{i, g_{i}}
\end{array}\right],
$$

where each $K_{i, k}$ is of dimension $m \times p_{i, k}$. For all odd $i \in\{1, \ldots, 2 \sigma\}$ and for each $i \in\{2 \sigma+1, \ldots, v\}$ and $k \in$ $\left\{1, \ldots, g_{i}\right\}$ we build vector chains of length $p_{i, k}$ as follows:

$$
\begin{aligned}
h_{i, k}(1) & =N_{i} K_{i, k}(1), \\
h_{i, k}(2) & =M_{i} \bar{\pi}\left\{h_{i, k}(1)\right\}+N_{i} K_{i, k}(2), \\
& \vdots \\
h_{i, k}\left(p_{i, k}\right) & =M_{i} \bar{\pi}\left\{h_{i, k}\left(p_{i, k}-1\right)\right\}+N_{i} K_{i, k}\left(p_{i, k}\right) .
\end{aligned}
$$

From these column vectors we construct matrices

$$
H_{i, k} \triangleq\left[h_{i, k}(1)\left|h_{i, k}(2)\right| \ldots \mid h_{i, k}\left(p_{i, k}\right)\right]
$$

of dimension $(n+m) \times p_{i, k}$, and

$$
H_{i} \triangleq \begin{cases}\mathfrak{R e}\left\{\left[H_{i, 1}|\ldots| H_{i, g_{i}}\right]\right\} & i \in\{1, \ldots, 2 \sigma\} \text { odd } \\ \mathfrak{I m}\left\{\left[H_{i-1,1}|\ldots| H_{i-1, g_{i}}\right]\right\} & i \in\{1, \ldots, 2 \sigma\} \text { even } \\ {\left[H_{i, 1}|\ldots| H_{i, g_{i}}\right]} & i \in\{2 \sigma+1, \ldots, v\}\end{cases}
$$

of dimension $(n+m) \times m_{i}$. Finally, we define real matrices

$$
\begin{aligned}
H_{K} & \triangleq\left[H_{1}|\ldots| H_{v}\right] \\
V_{K} & \triangleq \bar{\pi}\left\{H_{K}\right\} \\
W_{K} & \triangleq \underline{\pi}\left\{H_{K}\right\}
\end{aligned}
$$

of dimensions $(n+m) \times n, n \times n$ and $m \times n$, respectively. The dependence upon $K$ of the matrices defined in (12-14) has been made explicit. To guide the readers through these definitions, before presenting the parameterisation for the pole placing matrix $F$ in the main theorem of this paper, we illustrate the procedure of construction of the Jordan chains with the corresponding indices in a simple example. 
Example 2.1: Consider the reachable pair $(A, B)$ with

$$
A=\left[\begin{array}{cccc}
0 & 0 & 0 & -1 \\
0 & 0 & 0 & 0 \\
0 & -1 & 0 & 0 \\
0 & 0 & 0 & 0
\end{array}\right], \quad B=\left[\begin{array}{ll}
0 & 0 \\
0 & 1 \\
0 & 0 \\
1 & 0
\end{array}\right]
$$

We want to assign the closed loop eigenvalues $\lambda_{1}=-1-j$ and $\lambda_{2}=-1+j$ with double multiplicity, i.e., $\mathscr{L}=\{-1-$ $j,-1+j\}$ and $\mathscr{M}=\{2,2\}$. First, we notice that a basis matrix for the null-space of $S\left(\lambda_{1}\right)$ is given by the span of

$$
N_{1}=\left[\begin{array}{cc}
1 & 0 \\
0 & 2 \\
0 & 1-j \\
1+j & 0 \\
-2 j & 0 \\
0 & -2-2 j
\end{array}\right]
$$

and define $N_{2}=\bar{N}_{1}$. Moreover

$$
M_{1}=\frac{1}{7}\left[\begin{array}{cccc}
3-3 j & 0 & 0 & -2 j \\
0 & 2-2 j & -1 & 0 \\
0 & -2 j & 3-3 j & 0 \\
-1 & 0 & 0 & 2-2 j \\
1+j & 0 & 0 & 3 \\
0 & 3 & 1+j & 0
\end{array}\right]
$$

so that $M_{2}=\bar{M}_{1}$. By choosing for example the parameter matrix $K_{1,1}=\left[\begin{array}{cc}7 & 1 \\ -7 & 1\end{array}\right]$, we find

$$
\begin{aligned}
h_{1,1}(1) & =N_{1} K_{1,1}(1)=N_{1}\left[\begin{array}{c}
7 \\
-7
\end{array}\right] \\
& =7\left[\begin{array}{ccc}
1 & -2-1+j & 1+j-2 j 2+2 j
\end{array}\right]^{\mathrm{T}} \\
h_{1,1}(2) & =M_{1}\left[\begin{array}{c}
7 \\
-14 \\
7+7 i
\end{array}\right]+N_{1}\left[\begin{array}{l}
1 \\
1
\end{array}\right] \\
& =\left[\begin{array}{ccc}
6-5 j-1+3 j & 1+9 j 4+j 4+2 j-10-2 j
\end{array}\right]^{\mathrm{T}} .
\end{aligned}
$$

Hence, $H_{1,1}=\left[h_{1,1}(1) \mid h_{1,1}(2)\right]$. Moreover, $H_{1}=\mathfrak{R e}\left\{H_{1,1}\right\}$ and $H_{2}=\mathfrak{I m}\left\{H_{1,1}\right\}$, which yield

$V_{K}=\left[\begin{array}{cccc}7 & 6 & 0 & -5 \\ -14 & -1 & 0 & 3 \\ -7 & 1 & 7 & 9 \\ 7 & 4 & 7 & 1\end{array}\right], W_{K}=\left[\begin{array}{cccc}0 & 4 & -14 & 2 \\ 14 & -10 & 14 & -2\end{array}\right]$.

It is easily seen that $V_{K}$ is invertible. Again, a simple computation shows that the feedback matrix

$$
F_{K}=W_{K} V_{K}^{-1}=\frac{1}{41}\left[\begin{array}{cccc}
138 & -126 & 154 & -236 \\
-196 & 72 & -170 & 252
\end{array}\right]
$$

yields a closed-loop matrix $A+B F_{K}$ whose complex and real Jordan structures are respectively

$\left[\begin{array}{cc|cc}-1-j & 1 & 0 & 0 \\ 0 & -1-j & 0 & 0 \\ \hline 0 & 0 & -1+j & 1 \\ 0 & 0 & 0 & -1+j\end{array}\right]$ and $\left[\begin{array}{cc|cc}-1 & -1 & 1 & 0 \\ 1 & -1 & 0 & 1 \\ \hline 0 & 0 & -1 & -1 \\ 0 & 0 & 1 & -1\end{array}\right]$.

We are now ready to present the main result of this paper.

Theorem 2.1: For almost all choices of the parameter matrix $K$, matrix $V_{K}$ is invertible, i.e., $V_{K}$ is generically invertible for every choice of $K$ except possibly those laying in a proper algebraic variety. The set of all feedback matrices such that the Jordan structure of $A+B F$ is described by $\mathscr{L}, \mathscr{M}$ and $\mathscr{P}$ is parameterised in $K$ as

$$
F_{K}=W_{K} V_{K}^{-1}
$$

where $V_{K}$ and $W_{K}$ are obtained with a parameter matrix $K$ such that $V_{K}$ is invertible.

Proof: For the sake of simplicity and brevity, we only consider the case where $\mathscr{L}$ is real. We begin by showing that for any $V_{K}$ and $W_{K}$ in (13-14), and with feedback matrix given by (15), the closed-loop matrix has the desired eigenstructure, whenever $V_{K}$ is invertible. For each $i \in\{1, \ldots, v\}$, let $K_{i}$ be an input parameter matrix as in (7), and for each $k \in\left\{1, \ldots, g_{i}\right\}$, let $H_{i, k}$ be constructed as in (12). We may partition $H_{i, k}$, which is defined for all $i \in\{1, \ldots, v\}$, as

$$
H_{i, k}=\left[\begin{array}{ccc}
v_{i, k}(1) & \ldots & v_{i, k}\left(p_{i, k}\right) \\
w_{i, k}(1) & \ldots & w_{i, k}\left(p_{i, k}\right)
\end{array}\right],
$$

where the column vectors satisfy by construction

$$
\begin{aligned}
\left(A-\lambda_{i} I_{n}\right) v_{i, k}(1)+B w_{i, k}(1) & =0 \\
\left(A-\lambda_{i} I_{n}\right) v_{i, k}(2)+B w_{i, k}(2) & =v_{i, k}(1), \\
& \vdots \\
\left(A-\lambda_{i} I_{n}\right) v_{i, k}\left(p_{i, k}\right)+B w_{i, k}\left(p_{i, k}\right) & =v_{i, k}\left(p_{i, k}-1\right) .
\end{aligned}
$$

Define

$$
\begin{aligned}
V_{i, k} & =\left[v_{i, k}(1)\left|v_{i, k}(2)\right| \ldots \mid v_{i, k}\left(p_{i, k}\right)\right], \\
W_{i, k} & =\left[w_{i, k}(1)\left|w_{i, k}(2)\right| \ldots \mid w_{i, k}\left(p_{i, k}\right)\right],
\end{aligned}
$$

and also

$$
V_{i}=\left[V_{i, 1}|\ldots| V_{i, g_{i}}\right], \quad W_{i}=\left[W_{i, 1}|\ldots| W_{i, g_{i}}\right] .
$$

and $F_{K} V_{i}=W_{i}$ for all $i \in\{1, \ldots, v\}$. Hence, (17-19) can be written as

$$
\left(A+B F_{K}\right) V_{i}=V_{i} J\left(\lambda_{i}\right)
$$

or all $i \in\{1, \ldots, v\}$. Thus,

$$
\left(A+B F_{K}\right) X_{K}=X_{K} \Lambda
$$

where $X_{K}=\left[\begin{array}{llll}V_{1} & V_{2} & \ldots & V_{V}\end{array}\right]$ and $\Lambda$ as in (3), as required. We now show that for every feedback matrix $F$ that delivers a closed-loop matrix $A+B F$ whose eigenstructure is given by $\mathscr{L}, \mathscr{M}$ and $\mathscr{P}$, there exists a parameter matrix $K$ such that $F=W_{K} V_{K}^{-1}$, where $V_{K}$ and $W_{K}$ are computed from (13-14) with $V_{K}$ invertible. Let $\Lambda \triangleq A+B F$. Now, using a change of coordinates $T$ that brings $\Lambda$ into the Jordan canonical form, we can write

$$
(A+B F) T=T \underbrace{T^{-1} \Lambda T}_{\Lambda_{J}},
$$

where $\Lambda_{J}=\operatorname{diag}\left\{\Lambda_{1}, \ldots, \Lambda_{v}\right\}$ with $\Lambda_{i}=\lambda_{i}$ for all $i \in$ $\{1, \ldots, v\}$. Let $X=T$ and $Y=F T$. We find

$$
\left[\begin{array}{ll}
A & B
\end{array}\right]\left[\begin{array}{c}
X \\
Y
\end{array}\right]=X \Lambda_{J}
$$


Eq. (24) can be written as

$$
\begin{array}{r}
{\left[\begin{array}{ll}
A & B
\end{array}\right]\left[\begin{array}{c}
X \\
Y
\end{array}\right]=X \cdot \operatorname{diag}\left\{J_{1}\left(\lambda_{1}\right), \ldots, J_{g_{1}}\left(\lambda_{1}\right), J_{1}\left(\lambda_{2}\right), \ldots\right.} \\
\left.J_{g_{2}}\left(\lambda_{2}\right), \ldots, J_{1}\left(\lambda_{v}\right), \ldots, J_{g_{v}}\left(\lambda_{v}\right)\right\}
\end{array}
$$

where $g_{i}$ is the number of Jordan mini-blocks corresponding to the eigenvalue $\lambda_{i}$ and the generic Jordan mini-block $J_{k}\left(\lambda_{i}\right)$ is of order $p_{i, k}$. Let us partition $X$ and $Y$ conformably with the corresponding Jordan mini-blocks that they multiply, i.e.,

$$
\begin{aligned}
& {\left[\begin{array}{ll}
A & B
\end{array}\right]\left[\begin{array}{ccccccc}
X_{1,1} & \ldots & X_{1, g_{1}} & \ldots & X_{v, 1} & \ldots & X_{v, g_{v}} \\
Y_{1,1} & \ldots & Y_{1, g_{1}} & \ldots & Y_{v, 1} & \ldots & Y_{v, g_{v}}
\end{array}\right]=\left[X_{1,1} J_{1}\left(\lambda_{1}\right)\right.} \\
& \left.\ldots X_{1, g_{1}} J_{g_{1}}\left(\lambda_{1}\right) \ldots X_{1, g_{v}} J_{g_{v}}\left(\lambda_{1}\right) \ldots X_{v, g_{v}} J_{g_{v}}\left(\lambda_{v}\right)\right] .
\end{aligned}
$$

Consider the generic term of this product

$$
\left[\begin{array}{ll}
A & B
\end{array}\right]\left[\begin{array}{c}
X_{i, k} \\
Y_{i, k}
\end{array}\right]=X_{i, k} J_{k}\left(\lambda_{i}\right)
$$

where $J_{k}\left(\lambda_{i}\right)$ is the generic $k$-th Jordan mini-block relative to the eigenvalue $\lambda_{i}$. Partitioning $X_{i, k}=\left[\begin{array}{llll}v_{i, k, 1} & v_{i, k, 2} & \ldots & v_{i, k, p_{i, k}}\end{array}\right]$ and $Y_{i, k}=\left[\begin{array}{llll}w_{i, k, 1} & w_{i, k, 2} & \ldots & w_{i, k, p_{i, k}}\end{array}\right]$, we can write (25) as

$$
\begin{aligned}
& {\left[\begin{array}{ll}
A & B
\end{array}\right]\left[\begin{array}{cccc}
v_{i, k, 1} & v_{i, k, 2} & \ldots & v_{i, k, p_{i, k}} \\
w_{i, k, 1} & w_{i, k, 2} & \ldots & w_{i, k, p_{i, k}}
\end{array}\right]} \\
& \quad=\left[\begin{array}{llllll}
v_{i, k, 1} & v_{i, k, 2} & \ldots & v_{i, k, p_{i, k}}
\end{array}\right]\left[\begin{array}{ccccc}
\lambda_{i} & 1 & 0 & \ldots & 0 \\
0 & \lambda_{i} & 1 & \ldots & 0 \\
0 & 0 & \lambda_{i} & \ldots & 0 \\
\vdots & \vdots & \vdots & \ddots & \vdots \\
0 & 0 & 0 & \ldots & \lambda_{i}
\end{array}\right]
\end{aligned}
$$

which yields

$$
\begin{aligned}
& {\left[\begin{array}{lllll}
A v_{i, k, 1}+B w_{i, k, 1} & A v_{i, k, 2}+B w_{i, k, 2} & \ldots & A v_{i, k, p_{i, k}}+B w_{i, k, p_{i, k}}
\end{array}\right]} \\
& =\left[\begin{array}{llll}
v_{i, k, 1} \lambda_{i, k, 1} v_{i, k}+\lambda_{i} v_{i, k, 2} & \ldots & v_{i, k, p_{i, k}-1}+\lambda_{i} v_{i, k, p_{i, k}}
\end{array}\right] .
\end{aligned}
$$

Therefore, $\left[\begin{array}{l}v_{i, k, 1} \\ w_{i, k, 1}\end{array}\right] \in \operatorname{ker}\left[\begin{array}{rr}A-\lambda_{i} I_{n} & B \\ C & D\end{array}\right]$ implies that there exists $K_{i, k}(1)$ such that $\left[\begin{array}{l}v_{i, k, 1} \\ w_{i, k, 1}\end{array}\right]=N_{i} K_{i, k}(1)$. Moreover, from (26) we find

$$
\left[\begin{array}{ll}
A-\lambda_{i} I_{n} & B
\end{array}\right]\left[\begin{array}{c}
v_{i, k, 2} \\
w_{i, k, 2}
\end{array}\right]=v_{i, k, 1},
$$

which implies that there exists $K_{i, j}(2)$ such that

$$
\left[\begin{array}{c}
v_{i, k, 2} \\
w_{i, k, 2}
\end{array}\right]=M_{i} \bar{\pi}\left\{\left[\begin{array}{c}
v_{i, k, 1} \\
w_{i, k, 1}
\end{array}\right]\right\}+N_{i} K_{i, k}(2) .
$$

Repeating this procedure for all $l \in\left\{1, \ldots, p_{i, k}\right\}$, we find the parameters $K_{i, k}(1), \ldots, K_{i, k}\left(p_{i, k}\right)$ which satisfy (8)-(10) with $h_{i, k}(h)=\left[\begin{array}{l}v_{i, k, h} \\ w_{i, k, h}\end{array}\right]$. This procedure can be carried out for all Jordan mini-blocks. The fact that for almost every choice of the parameter matrix $K$, the matrix $V_{K}$ computed from (13) is non-singular follows from [14, Lemma 2.4].

\section{ILLUSTRATIVE EXAMPLES}

In this section, we compare the algorithm presented in this paper with the results obtained in Ait Rami et al. [9] and Linnemann [12].

Example 3.1: In Example 2.1, the method presented in this paper delivers the feedback matrix

$$
F=\left[\begin{array}{cccc}
2.0000 & 0.0000 & -0.0000 & -2.0000 \\
0.0000 & -2.0000 & 2.0000 & -0.0000
\end{array}\right],
$$

whose Frobenius norm is equal to 4 , whereas the algorithm in [9] yields

$$
F^{\prime}=\left[\begin{array}{cccc}
1.6346 & 0.0042 & 0.0480 & -1.9325 \\
-2.4959 & -2.0675 & 2.3738 & 0.9088
\end{array}\right],
$$

whose Frobenius norm is equal to 4.8346 . Another performance consideration is the accuracy of the pole placement achieved by each method. We use the measure

$$
\Delta(F) \triangleq \max \left\{\left|\operatorname{eig}_{i}(A+B F)-\lambda_{i}\right|: \lambda_{i} \in \mathscr{L}\right\},
$$

which represents the largest absolute value difference between each eigenvalue of $A+B F$, and the corresponding $\lambda_{i}$ in $\mathscr{L}$. In the present case we obtain $\Delta(F)=9.97 \times 10^{-13}$ and $\Delta\left(F^{\prime}\right)=2.47 \times 10^{-8}$. This result indicates that the method introduced in this paper achieves more accurate pole placement by some orders of magnitude.

Example 3.2: We consider the boiler system controller given in [13] with $n=9$ states and $m=4$ control inputs. In [12] a gain matrix is sought to place all the closed loop poles at $\lambda=-0.55$. The controllability indices for this example are $\{3,2,2,2\}$. Thus, this pole placement can be achieved with a largest Jordan block of dimensions of at least three, and at most 9 . Hence we may obtain a feedback matrix $F$ such that $(A+B F)^{l}=0$, for any $l$ between 3 and 9 .

For the case $(A-\lambda I+B F)^{3}=0$, in [12] the feedback matrix $F_{1}$ was reported with $\left\|F_{1}\right\|_{\text {FRO }}=1.5 \times 10^{7}$. Applying the method described in this paper, we obtain $F_{2}$ with $\left\|F_{2}\right\|_{\text {FRO }}=4.4 \times 10^{5}$.

For the case $(A-\lambda I+B F)^{5}=0$, [12] reported the solution $F_{3}$ with $\left\|F_{3}\right\|_{\mathrm{FRO}}=9.2 \times 10^{2}$. Using the procedure described here, we are able to obtain $F_{4}$ with $\left\|F_{4}\right\|_{\mathrm{FRO}}=6.8 \times 10^{2}$.

Finally, for the case $(A-\lambda I+B F)^{9}=0$, in [12] the feedback $F_{5}$ was obtained with $\left\|F_{5}\right\|_{\mathrm{FRO}}=2.8 \times 10^{5}$. Again, applying the method described here, we obtain $F_{6}$ with $\left\|F_{6}\right\|_{\text {FRO }}=1.6 \times 10^{1}$.

We see that in each case the method described in this paper obtains the desired eigenstructure with considerably reduced matrix gain than that of [12], by some orders of magnitude.

\section{CONCLUSION}

We have introduced a novel parametric form for the problem of exact pole placement that can accommodate any desired eigenstructure with arbitrary multiplicities. This method places no restrictions on the set of poles that can be assigned, or their multiplicities. The effectiveness of the 
method has been compared against that of the alternatives [9] and [12]. Examples were given to show that this method delivers pole placement that is substantially more accurate and with less matrix gain than these two methods. Future work will consider the application of the parametric form to achieving robust closed-loop eigenstructure that minimises sensitivity to uncertainties in the system matrices $(A, B)$, as well as the exploitation of the parameterisation to the end of achieving non-overshooting, non-undershooting and monotonic step response in the MIMO case, see e.g. [15][18].

\section{REFERENCES}

[1] H. H. Rosenbrock, State-Space and Multivariable Theory. New York: Wiley, 1970.

[2] J. Kautsky, J. N.K. Nichols and P. Van Dooren, Robust Pole Assignment in Linear State Feedback, International Journal of Control, vol. 41, pp. 1129-1155, 1985.

[3] R. Byers and S. G. Nash, Approaches to robust pole assignment, International Journal of Control, vol. 49, pp. 97-117, 1989.

[4] A. L. Tits and Y. Yang, Globally Convergent Algorithms for Robust Pole Assignment by State Feedback, IEEE Transactions on Automatic Control., vol. 41(10), pp. 1432-1452, 1996.

[5] R. Schmid, T. Nguyen and A. Pandey, Optimal Pole placement with Moore's algorithm, in Proceedings 1st IEEE Australian Control Conference (AUCC 2011), Melbourne, Australia, 2011.

[6] S.P. Bhattacharyya and E. de Souza, Pole assignment via Sylvester equation, Systems \& Control Letters, vol. 1(4), pp. 261-263, 1981.

[7] M.M. Fahmy and J. O'Reilly, Eigenstructure Assignment in Linear Multivariable Systems-A Parametric Solution, IEEE Transactions on Automatic Control, vol. 28, pp. 990-994, 1983.
[8] E. Chu, Pole assignment via the Schur form, Systems \& Control Letters vol. 56, pp, 303-314, 2007.

[9] M. Ait Rami, S.E. Faiz, A. Benzaouia, and F. Tadeo, Robust Exact Pole Placement via an LMI-Based Algorithm, IEEE Transactions on Automatic Control, Vol. 54(2) pp. 394-398, 2009.

[10] G. Klein and B.C. Moore, Eigenvalue-Generalized Eigenvector Assignment with State Feedback, IEEE Transactions on Automatic Control, vol. 22(1), pp. 141-142, 1977.

[11] H.K. Tam and J. Lam, Newton's approach to gain-controlled robust pole placement, IEE Proc.-Control Theory Applications. Vol. 144(5), pp. 439-446, 1997.

[12] A. Linnemann, An algorithm to compute state feedback matrices for multi-input deadbeat control, Systems \& Control Letters vol. 25, pp, 99-102, 1995.

[13] A. Grace, A.J Laub, J.N. Little, C.MF. Thompson, Control Systems Toolbox for Use with MATLAB, The Mathworks, 1992.

[14] P.J. Antsaklis and Z. Gao, Polynomial and rational interpolation: theory and control applications, International Journal of Control, vol. 58(2), pp. 349-404, 1993.

[15] R. Schmid, and L. Ntogramatzidis, On the design of non-overshooting linear tracking controllers for right invertible systems. In Proceedings of the $48^{\text {th }}$ Conference on Decision and Control (CDC 09), Shanghai, P.R. China, Dec. 16-18, 2009.

[16] R. Schmid, and L. Ntogramatzidis, Achieving a nonovershooting transient response with multivariable dynamic output feedback tracking controllers. In Proceedings of the $48^{\text {th }}$ Conference on Decision and Control (CDC 09), Shanghai, P.R. China, Dec. 16-18, 2009.

[17] R. Schmid, and L. Ntogramatzidis, A unified method for the design of nonovershooting linear multivariable state-feedback tracking controllers. Automatica, 46: 312-321, 2010.

[18] R. Schmid, and L. Ntogramatzidis, The design of nonovershooting and nonundershooting multivariable state feedback tracking controllers. Systems \& Control Letters, 61:714-722, 2012. 